\title{
Distribución Volumétrica, Simetría del ChorRo y Diámetro de Gotas DE LAS Boquillas TF-VS2 ${ }^{1}$
}

\author{
Spraying Distribution, Symmetry of Fan and Droplet Size to Spray Nozzles TF-VS2 \\ ROMÁN, R.A.A. ${ }^{2}$, FERREIRA, M.C. ${ }^{3}$, CARVALHO, G.F.G. ${ }^{4}$ y BAGGIO, M.V. ${ }^{5}$
}

\begin{abstract}
RESUMEN - E1 conocimiento de las características de las diferentes boquillas de pulverización tiene una importancia imprescindible para la adecuada y correcta recomendación de su uso. El objetivo de este trabajo fue determinar el espaciamiento máximo en la barra horizontal de pulverización, la simetría del chorro y el diámetro de gotas en boquillas de pulverización TF-VS2. E1 trabajo fue realizado en mesa de deposición, en donde fueron utilizadas las presiones de 100, 200 y $300 \mathrm{kPa}$ en las alturas de trabajo de 40 y $50 \mathrm{~cm}$. La simetría del chorro fue determinada en función de dos metodologias (empírica y trigonométrica). También se determinó el tamaño de las gotas, utilizando el método de difracción de rayos laser, en función de dos caldos de pulverización constituidos por agua y por agua con adyuvante en las tres presiones de trabajo ya descritas. Los espaciamientos máximos entre las boquillas en la barra de pulverización no pueden rebasar los 70 y $82 \mathrm{~cm}$, admitiéndose el CV de un $10 \%$ para las alturas de 40 y de $50 \mathrm{~cm}$ respectivamente. La mayor presión proporcionó el menor diámetro mediano volumétrico (DMV) y la peor uniformidad de gotas, además del mayor porcentaje de gotas susceptibles a deriva, así como también aumentó la simetría entre los chorros.
\end{abstract}

Palabras-clave: tecnología de aplicación, tamaño de gotas, pulverizador.

\begin{abstract}
The knowledge of spray distribution pattern and analysis of droplets size of spray hydraulic nozzles turbo Floodjet TF-VS2 to adapt the spacing on spray boom are very important for a proper and correct use and recommendations. The aim of this study was to determine the maximum spacing on the horizontal spraying boom held on a patternator to the tips TF-VS2, under pressures of 100, 200 and $300 \mathrm{kPa}$ in heights of work of 40 and $50 \mathrm{~cm}$. Symmetry of spraying fan was determined due empirical and trigonometric methodology. It was also determined the droplets size by means of laser diffraction, with two spray liquids consisting of water and water plus adjuvant under three work pressures, described above. The maximum spacing between nozzles should be 70 and $82 \mathrm{~cm}$ assuming the coefficient of variation of $10 \%$ for the heights of 40 and $50 \mathrm{~cm}$ respectively. The higher pressure of work provided the lowest volume median diameter (VMD) and the worst uniformity of drops, beyond the higher percentage of drops susceptible to drift and improve the spray symmetry.
\end{abstract}

Keywords: spraying technology, droplet size, sprayer.

\section{INTRODUCCIÓN}

Gran parte de los perjuicios en las distintas culturas se debe a factores bióticos, los cuales se puede manejar con la utilización del control químico. Este método depende de la tecnología de aplicación de fitosanitarios para que haya uniformidad en la distribución del producto, reducción de la deriva, menor coste de la aplicación y menor contaminación ambiental (Ramos et al., 2006).

Entre los factores bióticos que influencian el desarrollo y la productividad de diversas

1 Recebido para publicação em 5.12.2009 e na forma revisada em 12.11.2010.

2 Engo ${ }^{0}$-Agr ${ }^{0}$., M.Sc., Dep. Fitossanidade, Universidade Estadual Paulista “Júlio de Mesquita Filho"- UNESP, 14884-900 JaboticabalSP, <alandiaroman@gmail.com>; ${ }^{3}$ Professor, Dr., Dep. de Fitossanidade, UNESP,Jaboticabal-SP; ${ }^{4}$ Eng ${ }^{\circ}$.-Agr ${ }^{\circ}$., Doctorando do Dep. de Fitossanidade, UNESP, Jaboticabal-SP, ${ }^{5}$ Biol., M.Sc., Dep. de Fitossanidade, UNESP, Jaboticabal-SP. 
culturas, como por ejemplo, de la caña de azúcar (Kuva et al., 2003), se puede citar las malas hierbas. Para el control de éstas, son indicadas boquillas de impacto o espejo, las cuales pueden operar a bajas presiones y en pequeñas distancias del blanco biológico, esto debido a una mayor apertura del ángulo del chorro. Por producir gotas grandes, hay una reducción de la deriva. Sin embargo, cuando es utilizado a altas presiones, hay la formación de gotas menores, lo que puede aumentar el porcentaje de gotas susceptibles al arrastre por el viento (Matuo, 1990).

Las gotas formadas por las boquillas de pulverización pueden tener su tamaño alterado por la presión de trabajo, formulación del producto utilizado, tipo y conformación de la boquilla. Estos últimos son los responsables de la calidad de la aplicación, considerando que interferirán sobre el caudal, cobertura del blanco y en la uniformidad de distribución del caldo (Bauer \& Raetano, 2004; Fernandes et al., 2007).

Además de esto, para la aplicación de productos fitosanitarios, el equipo a ser utilizado deberá estar regulado y calibrado, para que haya una mayor uniformidad de distribución volumétrica. La mayor parte de los aplicadores no se preocupa con ajustar el espaciamiento entre boquillas, la altura de la barra ni con eligir correctamente el tipo de boquilla que deberá ser utilizada. De esta manera, ocurre el desperdicio del producto que será aplicado, y aumenta la deriva o escurrimiento del mismo en las plantas (Ramos et al., 2006).

Para determinar el estándar de distribución de caldo, es utilizado el coeficiente de variación, lo cual se determina a partir de boquillas de pulverización individuales. Luego, se hace la simulación para la sobreposición de chorros de las boquillas, para determinar el espaciamiento más adecuado entre ellas. Son aceptables CV inferiores al 10\%, de acuerdo con los parámetros brasileños e inferiores al $7 \%$, de acuerdo con las normas europeas (Bauer et al., 2006; Cunha et al., 2004; Freitas et al., 2005;). Con relación a la determinación del diámetro de gotas, este puede ser realizado por medio de la técnica de difracción de rayos láser, que varía de acuerdo con las condiciones operacionales (Cunha et al., 2007).

Hay en el mercado diversos tipos de boquillas para distintas finalidades. Para el control de las malas hierbas en el cultivo de la caña de azúcar, se puede utilizar boquillas de chorro plano de ángulo grande Turbo Floodjet, que está indicada para la aplicación de herbicida en preemergencia, pues produce gotas mayores y menor deriva (Matuo, 1990).

Es necesario determinar el coeficiente de variación para este modelo de boquilla para evitar la sobreposición excesiva entre boquillas. De esta manera, se reduce la pérdida del producto y el coste de la aplicación. Además, el análisis del tamaño de gotas también es importante para determinar la mejor presión de trabajo, que irá proporcionar el tamaño de gota más adecuado para alcanzar el blanco con mejor calidad.

E1 objetivo de este trabajo fue el de determinar el espaciamiento máximo en la barra horizontal de pulverización para la boquilla TF-VS2 en función de tres presiones y de dos alturas de trabajo, así como determinar el tamaño de gotas en función de dos caldos y tres presiones de trabajo y también la simetría entre los chorros aspergidos.

\section{MATERIAL Y MÉTODOS}

E1 presente trabajo fue realizado en el departamento de Fitosanidad de la UNESP, Campus de Jaboticabal. E1 estudio utilizó boquillas hidráulicas Turbo Floodjet TF-VS2 (Boquilla de acero inoxidable de chorro plano con ángulo grande y gotas extremamente gruesas, conforme instrucciones del fabricante), en las presiones de 100, 200 y $300 \mathrm{kPa}$. E1 sistema hidráulico fue montado con el auxilio de aire comprimido para que los análisis pudieran ser realizados a presión constante.

\section{Estudio de la distribución de volumen en mesa de evaluación}

En la Unidade de Investigación y Enseñanza de Técnicas de Aplicación de Defensivos Agrícolas ("Pesquisa e Ensino de Técnicas de Aplicação de Defensivos Agrícolas"), se evaluó el perfil de distribución volumétrica 
de caldo en mesa de deposición metálica, formada por 67 canales distantes $2,5 \mathrm{~cm}$ uno del otro que conducian el caldo hasta que fuera acondicionado en tubos graduados para la posterior lectura del volumen. Las tres presiones elegidas para el análisis $(100,200$ y $300 \mathrm{kPa}$ ) fueron evaluadas en dos alturas de pulverización de las boquillas, 40 y $50 \mathrm{~cm}$. La boquilla fue posicionada en la tercera canaleta debido al hecho de que aquella proporciona una gran abertura de ángulo a partir de $100 \mathrm{kPa}$. Por lo tanto, fue medida la primera mitad de la proyección del chorro y posteriormente la otra mitad. En las primeras lecturas de cada tratamiento, se mantuvo el sistema hidráulico en funcionamiento hasta que alguno de los tubos alcanzara $90 \%$ de su volumen. Para una mejor visualización, se controló el tiempo para la realización de las repeticiones. En todas las aplicaciones, los caldos fueron constituidos por agua. A partir de eso, fueron calculados los perfiles de deposición y, con el auxilio del software Microsoft Excel ${ }^{\circledR}$, fueron calculados los valores de coeficiente de variación (CV) de la sobreposición entre los chorros pulverizados, por medio de la simulación de espaciamiento entre boquillas de 40, 50, 60, 70 y $80 \mathrm{~cm}$. Para determinar el espaciamiento entre boquillas de forma que representaran una adecuada recomendación, se consideró el valor de coeficiente de variación máximo del 10\%, conforme Wolf \& Smith (1979) y FAO (1997). Tras la obtención de los valores en la mesa de distribución, fue estudiada la simetría entre los chorros por la boquilla de pulverización en estudio, por medio de regresión linear.

Los ángulos de apertura del chorro fueron medidos de dos maneras: de forma empírica con la utilización de goniómetro; y por medio del cálculo, utilizando la función trigonométrica del arco tangente según indica Viana et al. (2007) y Camara et al. (2008). Los caudales de las boquillas fueron medidos en cada repetición con el tiempo de colecta de un minuto. Para constatar la diferencia entre ambas metodologías de evaluación de ángulo, los promedios de las lecturas fueron analizados estadísticamente y comparados por medio del test de Tukey al 5\% de probabilidad.

\section{Determinación del diámetro de gotas}

En el Laboratorio de Análisis de Tamaño de Partículas (Laboratório de Análise do
Tamanho de Partículas (LAPAR)), se determinó el espectro de la población de gotas a partir del DMV - diámetro de gota, considerando que el $50 \%$ del volumen de líquido pulverizado está constituido por gotas de tamaño mayor o menor que este valor, amplitud relativa y el porcentual de gotas menor que $100 \mu \mathrm{m}$, para determinar así la cantidad de gotas susceptibles a deriva. Las mezclas utilizadas en este estudio eran a base de agua o agua y Haiten en una concentración de un $0,1 \%$. Fue utilizado un analizador en tiempo real con a base de rayo láser que sufre difracción durante el pasaje de las gotas pulverizadas por la región de muestreo del aparato. El equipamiento dispone de unidad óptica que detecta el estándar de difracción de la luz al atravesar un conjunto de partículas. La deviación que el laser sufre depende del tamaño de la partícula. Cuanto menor la partícula, mayor es el grado de difracción que el rayo de luz sufre (Fernandes et al., 2007). Se utilizó el equipamiento Mastersizer S, versión 2.19, de la Malvern Instruments Ltd. Los promedios fueron analizados con la ayuda del software ESTAT, con el cual se realizó el test de Tukey a un $5 \%$ de probabilidad.

\section{RESULTADOS Y DISCUSIÓN}

\section{Estudio de la distribución de volumen en mesa de evaluación}

En la Figura 1, se presenta las curvas de deposición para las presiones de 100, 200 y $300 \mathrm{kPa}$ en las alturas de 40 y $50 \mathrm{~cm}$ con relación a la superficie de recepción de caldo.

E1 perfil de distribución de la boquilla TF-VS2 en la altura de $40 \mathrm{~cm}$ y presión de $100 \mathrm{kPa}$ presenta una depresión del volumen colectado entre las probetas 10 a 15 aproximadamente, lo que indica un defecto de la boquilla de pulverización cuando trabajada a bajas presiones. Por otra parte, con las presiones de 200 y $300 \mathrm{kPa}$, esta depresión no apareció, presentando perfiles de distribución dentro de los estándares recomendados por el fabricante (Spraying Systems, 2003). Esto ratifica los resultados obtenidos por Perecin $\&$ Peressin (2003), que, evaluando los estándares de distribución para las boquillas $\mathrm{XR}, \mathrm{TJ}, \mathrm{TF}$, TQ, FL y TK, concluyeron que las boquillas XR, TF y FL presentaron los mejores estándares de distribución.

Planta Daninha, Viçosa-MG, v. 28, n. 4, p. 897-905, 2010 
En la altura de $50 \mathrm{~cm}$, se puede observar que para la presión de $100 \mathrm{kPa}$ no hubo uniformidad en el perfil de distribución, lo que indica que el modelo de la boquilla utilizada no es adecuado para trabajar a esta presión o a menores (Figura 1). En la presión de $200 \mathrm{kPa}$, el perfil de distribución fue más homogéneo y con el máximo de volumen en el centro del chorro, decreciendo en las extremidades. En general, los defectos de las boquillas evaluadas se repiten en ambas alturas, así como su comportamiento con relación a la distribución volumétrica. El trabajo en mayores alturas posibilita un mayor ancho de pulverización, faja de distribución de liquido, como se puede observar cuando los gráficos son comparados en ambas alturas, en el que en la altura de 50 cm las 96 canaletas utilizadas son casi completadas en su totalidad, lo que no ocurre cuando el trabajo es realizado a $40 \mathrm{~cm}$ de altura. Este hecho también fue verificado por Bauer \& Raetano (2004), que, trabajando en la evaluación de la distribución volumétrica de boquillas XR, TP y TJ, verificaron un incremento del 10\% en el ancho de deposición con el aumento de altura de barra de 40 para $50 \mathrm{~cm}$.

En la Figura 2, se presenta los valores de coeficiente de variación para los espaciamientos simulados $(50,60,70$, y $80 \mathrm{~cm})$. En la altura de $40 \mathrm{~cm}$, asumiendo el valor de hasta el $10 \%$ para los coeficientes de variación, los espaciamientos máximos para las presiones de 100, 200 y $300 \mathrm{kPa}$ fueron 69,9; 70 y $63 \mathrm{~cm}$ respectivamente, con mejor uniformidad en la barra horizontal de pulverización, obtenién dose así un margen de seguridad para las variaciones en campo generadas por condiciones de aplicación y por las oscilaciones constantes de la barra (Nation, 1982). En condiciones de campo, se puede observar aplicaciones con espaciamientos entre boquillas de $150 \mathrm{~cm}$. En este caso, el criterio
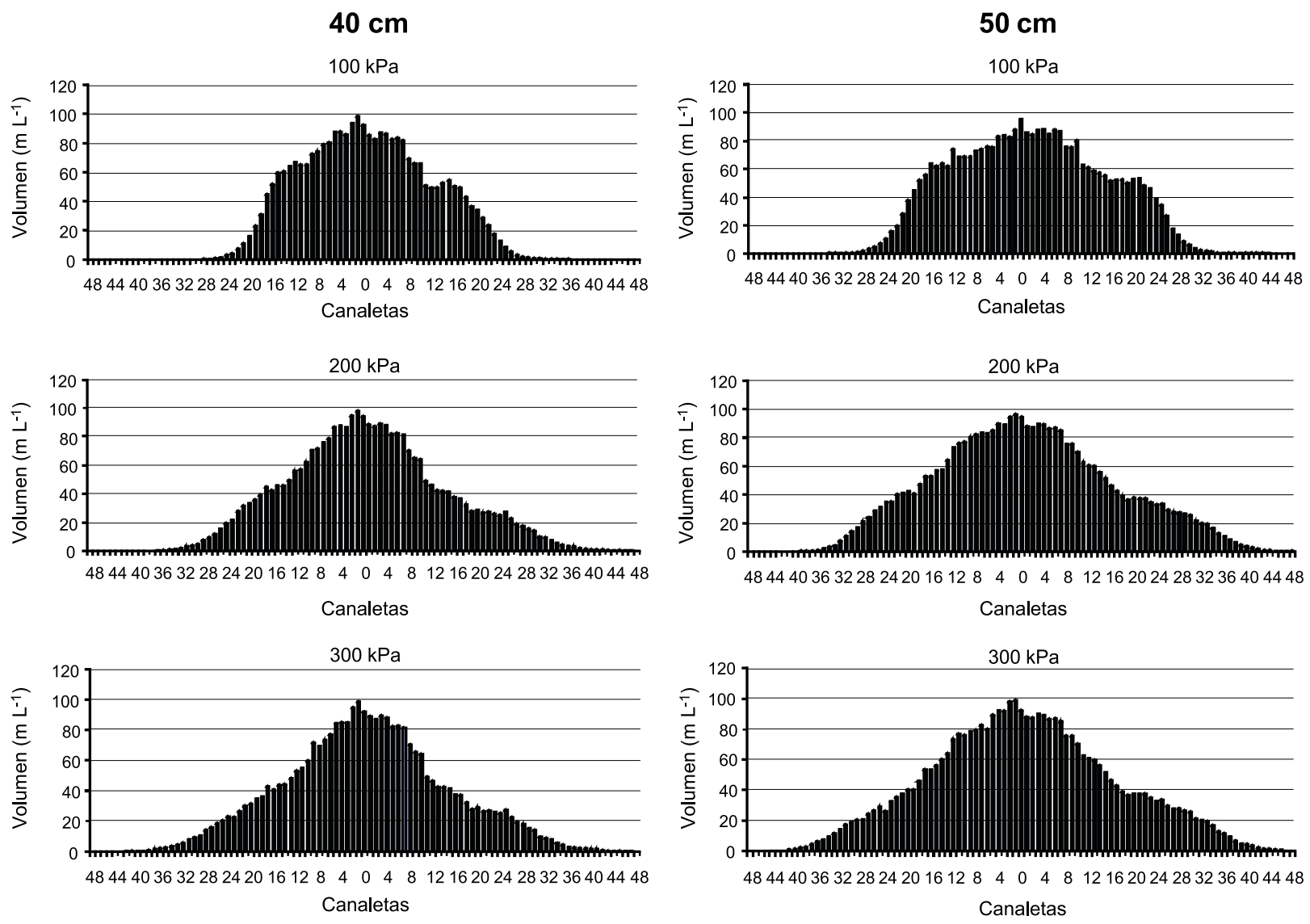

Figura 1 - Perfil de distribución volumétrica de la boquilla de pulverización TF-VS2 en las presiones de 100, 200 y 300 kPa en las alturas de 40 y $50 \mathrm{~cm}$. 
adoptado es el empírico, pues, de acuerdo con este estudio, se observa que el espaciamiento máximo para obtener $\mathrm{CV}$ aceptable es de $70 \mathrm{~cm}$. Por medio de una simulación, se pudo observar que, con espaciamiento de $150 \mathrm{~cm}$ entre boquillas en la barra, el CV alcanzó el $73 \%$, lo que resulta un alto riesgo de fallos en la aplicación. Galli et al. (1983) ya habian señalado la necesidad de estudios de las boquillas de pulverización antes de su utilización, principalmente para evitar errores en la aplicación producidos por la falta de uniformidad de distribución volumétrica.

En la altura de $50 \mathrm{~cm}$, los valores de los espaciamientos máximos aceptables para las presiones de 100, 200 y $300 \mathrm{kPa}$ fueron 75,59; 79,52 y $82,14 \mathrm{~cm}$ respectivamente. De acuerdo con el fabricante, los espaciamientos recomendados son de 50, 75 y $100 \mathrm{~cm}$ (Teejet, 2008). Si estos valores no obedecieran a las alturas de trabajo recomendadas $(60,75$ y $100 \mathrm{~cm})$, los
$\mathrm{CV}$ en trabajo con altura de $50 \mathrm{~cm}$ serían de 10,$36 ; 9 ; 69$ y $35 \%$ respectivamente, para la presión de $100 \mathrm{kPa}, 3,76 ; 6,91$ y 5,43 en la presión de $200 \mathrm{kPa}$ y 2,$58 ; 7,28$ y 19,48 en la presión de $300 \mathrm{kPa}$. Lo que muestra que sólo en la presión de $200 \mathrm{kPa}$ la uniformidad de pulverización se mantiene aceptable con altura de barra de $50 \mathrm{~cm}$ para los espaciamientos recomendados por el fabricante (Figura 2).

En la Figura 3, se presenta los gráficos de simetría entre los chorros pulverizados. Se puede observar en la altura de $40 \mathrm{~cm}$, para las tres presiones, coeficientes de determinación superiores a 0,96 , que es un indicativo de simetría entre los chorros. Ferreira et al. (2007), en un estudio realizado con boquillas de pulverización modelo ADGA 110015, afirman que, en las presiones de 207 y $310 \mathrm{kPa}$, los coeficientes de determinación superiores a 0,96 son indicativo de simetría. En la altura de $50 \mathrm{~cm}$, no se constató lo mismo en la presión
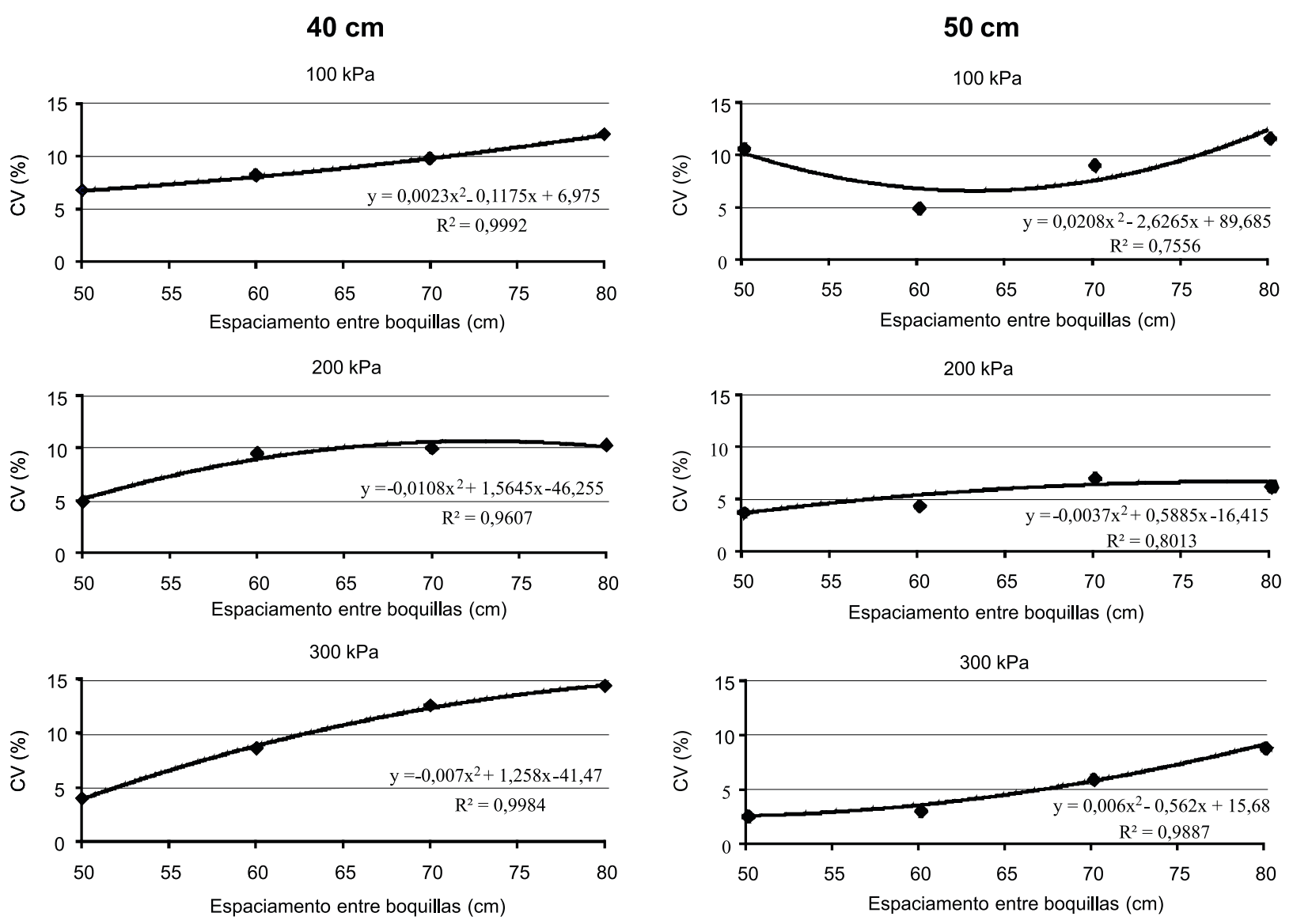

Figura 2 - Coeficientes de variación de la simulación del espaciamiento entre boquillas en la barra, en las presiones de 100,200 y $300 \mathrm{kPa}$ en las alturas de 40 y $50 \mathrm{~cm}$. 
$40 \mathrm{~cm}$
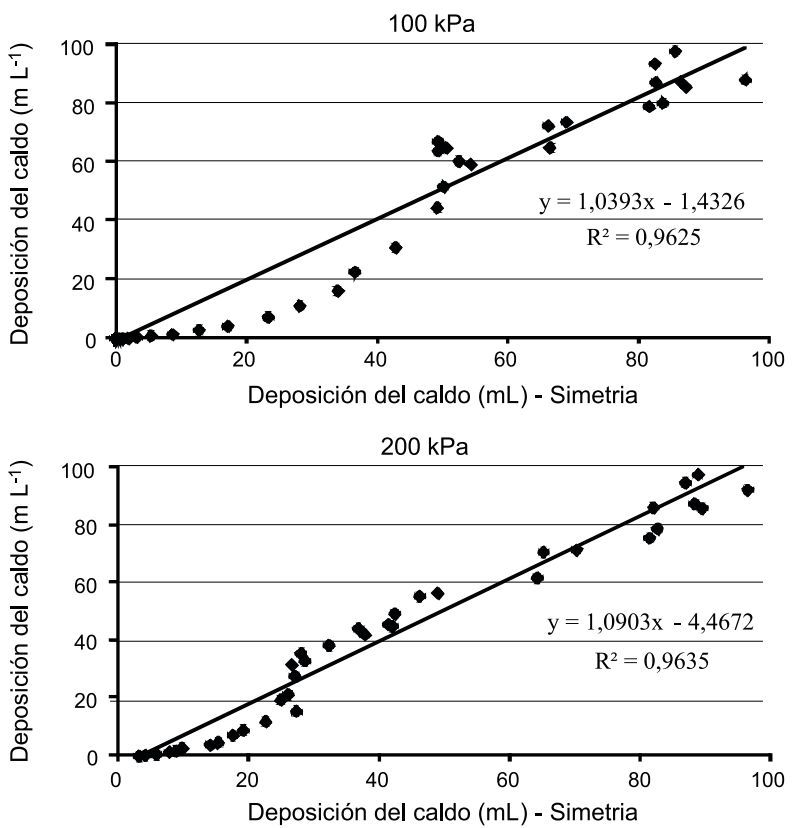

$300 \mathrm{kPa}$

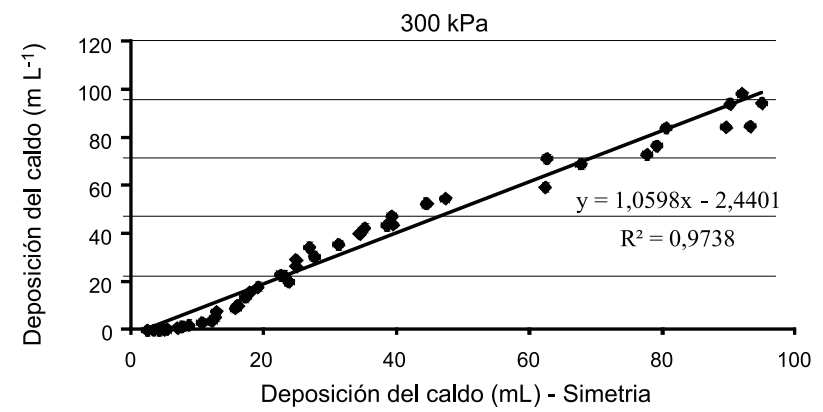

$50 \mathrm{~cm}$
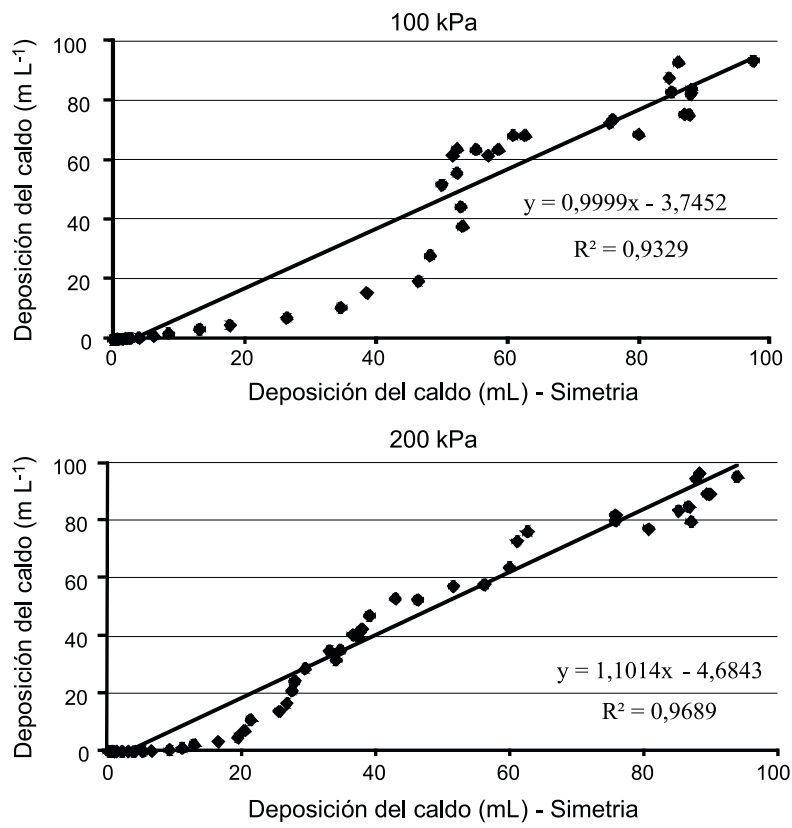

$300 \mathrm{kPa}$

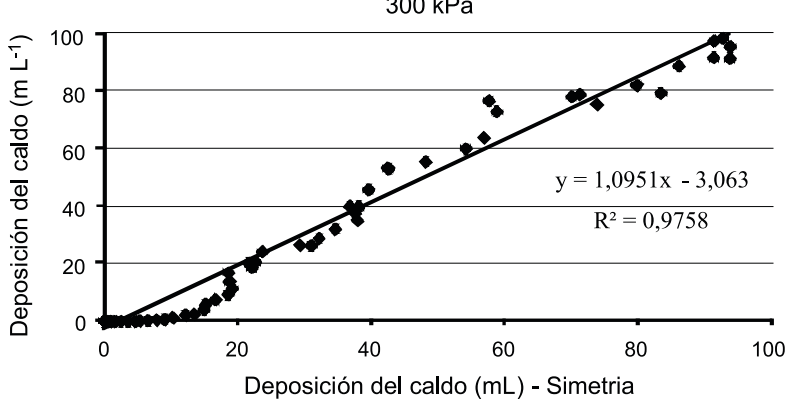

Figura 3 - Simetría de los chorros rociados en dos alturas y tres presiones de trabajo.

de $100 \mathrm{kPa}$, para la cual el coeficiente de determinación fue inferior a 0,96 , sin embargo se haya mantenido superior a 0,9. Esto probablemente se debe al hecho de que la presión mínima recomendada no presenta energía suficiente para mantener el mismo grado de simetría para ambos lados del chorro. En las presiones de 200 y $300 \mathrm{kPa}$, los chorros pueden ser considerados simétricos.

En la Tabla 1, se presenta los datos de angulación calculada, medida y del caudal nominal de las boquillas TF-VS2 en las tres presiones $(100,200$ y $300 \mathrm{kPa})$ y dos alturas (40 y $50 \mathrm{~cm}$ ) evaluadas.

Tanto para el ángulo calculado como para el medido empíricamente, se pudo observar el aumento de la angulación del chorro en función del aumento de la presión, en todas las alturas de trabajo. Cuando se compara las metodologías, hay una diferencia significativa al nivel del $5 \%$ de probabilidad por el test de Tukey. Se observó que el ángulo del chorro en la altura de $50 \mathrm{~cm}$ en las tres presiones de trabajo es mayor con la relación a la altura de $40 \mathrm{~cm}$. Esto ocurre debido a que, cuando se utiliza la formula trigonométrica, se considera los catetos, tanto adyacentes como opuestos. En el caso del cateto opuesto, este es mayor en la altura de $50 \mathrm{~cm}$, a causa del incremento de la deposición en alturas mayores (Bauer \& Raetano, 2004). De esta forma, el cateto opuesto, puesto que es numerador, irá interferir directamente sobre los resultados 
Tabla 1 - Angulo calculado, medido y caudal nominal de las boquillas TF-VS2 en función de la presión y altura de trabajo

\begin{tabular}{|c|c|c|c|}
\hline $40 \mathrm{~cm}$ & Ángulo calculado $\left({ }^{\circ}\right)$ & Ángulo medido $\left({ }^{\circ}\right)$ & Caudal (L.min $\left.{ }^{-1}\right)$ \\
\hline $100 \mathrm{kPa}$ & $126,87 \mathrm{a}$ & $115 \mathrm{~b}$ & 0,80 \\
\hline $200 \mathrm{kPa}$ & $128,65 \mathrm{a}$ & $135 \mathrm{~b}$ & 1,23 \\
\hline $300 \mathrm{kPa}$ & $159,61 \mathrm{a}$ & $145 \mathrm{~b}$ & 1,50 \\
\hline $50 \mathrm{~cm}$ & Ángulo calculado ( $\left.{ }^{\circ}\right)$ & Ángulo medido $\left({ }^{\circ}\right)$ & Aumento de caudal (\%) \\
\hline $100 \mathrm{kPa}$ & $151,24 \mathrm{a}$ & $119 \mathrm{~b}$ & 0 \\
\hline $200 \mathrm{kPa}$ & $154,11 \mathrm{a}$ & $139 \mathrm{~b}$ & 35 \\
\hline $300 \mathrm{kPa}$ & $155,47 \mathrm{a}$ & $146 \mathrm{~b}$ & 18 \\
\hline
\end{tabular}

Los promedios seguidos de la misma letra minúscula en la línea no son distintos, considerando el test de Tukey al 5\%.

mayores. Es importante señalar la pérdida de velocidad en las extremidades del chorro, lo que hace que el ancho de deposición sea menor, influenciando el resultado del ángulo calculado. Midiendo el ángulo con un goniómetro, valores mayores que los calculados y más próximos del indicado por el fabricante deberían ser observados, debido a que no se considera la pérdida de velocidad del chorro en el final del curso. Sin embargo, esto no ocurre, lo que hace que este método de medición tenga baja exactitud y, por lo tanto, sea susceptible a errores.

Con relación al caudal de las boquillas (Tabla 1), en las tres presiones de trabajo, las boquillas se encuentran dentro de los estándares de utilización respecto a los indicados por el fabricante. Con el aumento de la presión de 100 para $200 \mathrm{kPa}$, se puede observar un incremento de un $35 \%$ en el caudal, pues considerando el incremento de 200 para $300 \mathrm{kPa}$, el porcentual de incremento es de un $18 \%$.

\section{Determinación del diámetro de gotas}

En la Tabla 2, se presenta los resultados del análisis para los valores de DMV, Amplitud Relativa y porcentaje de gotas menores que $100 \mu \mathrm{m}$.

Se puede observar que la adición de adyuvante al caldo ocasionó disminución significativa para el DMV (Tabla 2), pero no alteró la amplitud relativa y el porcentaje de gotas menores que $100 \mu \mathrm{m}$. Para las presiones de trabajo, el aumento ocasionó la disminución del tamaño de gotas, con diferencia significativa entre las presiones evaluadas, ratificando los resultados obtenidos por Cunha et al. (2007). Esto se debe debido al aumento de fuerza ejercida en el orificio de salida de la boquilla que resulta una mayor fragmentación de las gotas, haciendo con que se reduzcan. La disminución del tamaño de gotas, se refleja directamente en la susceptibilidad de las mismas a las condiciones adversas de humedad relativa, viento y temperatura. De acuerdo con el fabricante, las gotas de esta boquilla son clasificadas como extremamente gruesas, o sea, presentan baja susceptibilidad a deriva. Según los resultados, incluso en la menor presión, el DMV de las gotas puede ser clasificado como de gotas medianas y el riesgo de deriva es igualmente bajo. La uniformidad de las gotas presentó diferencias significativas,

Tabla 2 - Diámetro Mediano Volumétrico (DMV), Amplitud Relativa (A.R) y porcentaje de gotas menores que $100 \mu \mathrm{m}$ en función de tres presiones de trabajo

\begin{tabular}{|c|c|c|c|}
\hline Caldo & DMV* & A.R & $<100 \mu \mathrm{m}$ \\
\hline Agua & $305,66 \mathrm{a}$ & $14,4 \mathrm{a}$ & $6,57 \mathrm{a}$ \\
\hline Haiten & $293,65 \mathrm{~b}$ & $8,5 \mathrm{a}$ & $6,54 \mathrm{a}$ \\
\hline Presiones $(\mathrm{kPa})$ & & & \\
\hline 100 & $371,88 \mathrm{a}$ & $7,9 \mathrm{a}$ & $2,78 \mathrm{a}$ \\
\hline 200 & $279,40 \mathrm{~b}$ & $7,4 \mathrm{~b}$ & $6,62 \mathrm{~b}$ \\
\hline 300 & $247,68 \mathrm{c}$ & $18,8 \mathrm{c}$ & $9,81 \mathrm{c}$ \\
\hline Test de $\mathrm{F}$ & & & \\
\hline C & $8,78^{* *}$ & $1,89^{\text {ns }}$ & $1,77^{\text {ns }}$ \\
\hline $\mathrm{P}$ & $337,86^{* *}$ & $304,46^{\mathrm{ns}}$ & $259,84^{* *}$ \\
\hline C x P & $0,86^{\mathrm{ns}}$ & $3,35^{*}$ & $0,15^{\mathrm{ns}}$ \\
\hline Promedio & 299,65 & 1,57 & 6,41 \\
\hline CV $(\%)$ & 4,06 & 2,77 & 11,80 \\
\hline
\end{tabular}

Los promedios seguidos por la misma letra minúscula en la columna no son distintos, considerando el test de Tukey.

* DMV: Diámetro Mediano Volumétrico; A.R.: Amplitud Relativa 


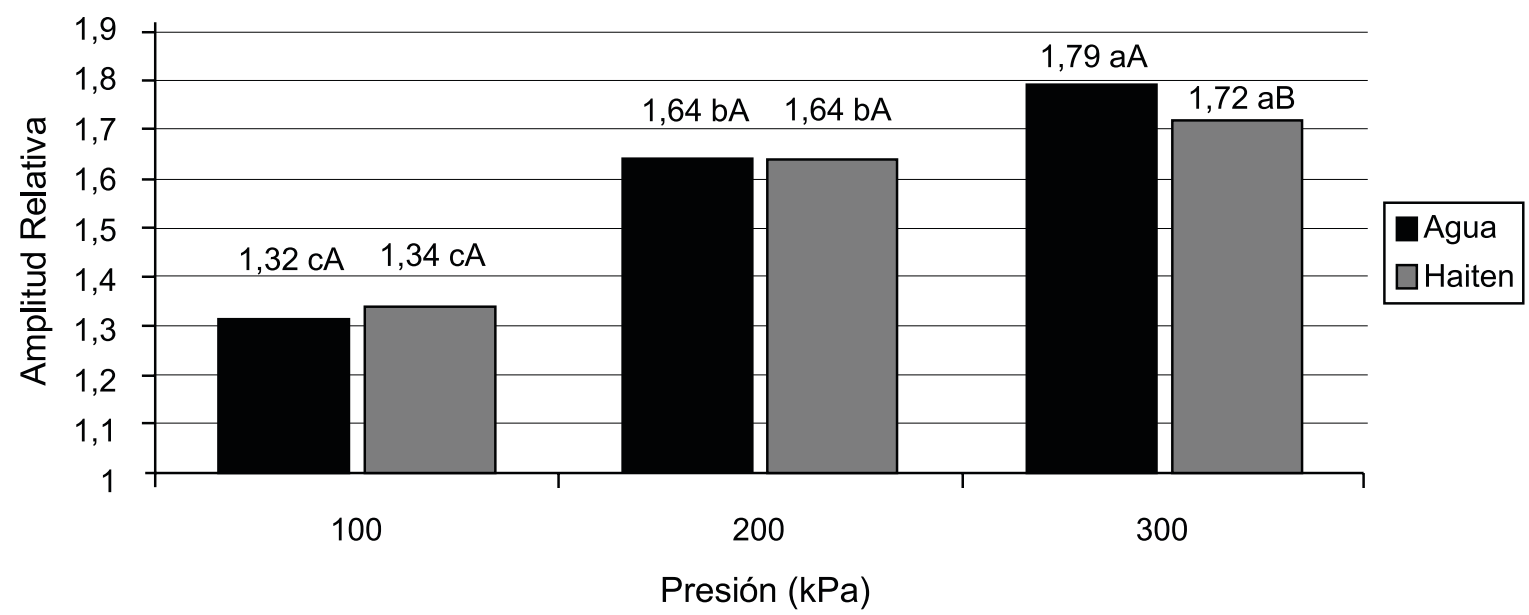

Los promedios seguidos de la misma letra minúscula entre caldos y mayúscula entre presiones no son distintos, considerando el test de Tukey al 5\% de probabilidad.

Figura 4 - Desdoblamiento de la interacción entre los factores caldo y presión para el parámetro de evaluación Amplitud Relativa del espectro de gotas.

con mayor uniformidad en la presión de $100 \mathrm{kPa}$. Ferreira et al. (2007) y Fernandes et al. (2007), trabajando con presiones de 200 y $300 \mathrm{kPa}$ con boquillas ADGA y XR respectivamente, no observaron diferencias significativas en el aumento de la presión de trabajo. En este parámetro, hubo interacción entre los factores en los cuales los valores son presentados en la Figura 4. Se verificó, a causa del desdoblamiento, que no hubo diferencia significativa al añadir el adyuvante con relación al agua para el porcentaje de gotas menores que $100 \mu \mathrm{m}$ (Figura 4). Los valores obtenidos de este parámetro refuerzan la confiabilidad de este modelo de boquilla para posibilidad de trabajos en condiciones de humedad relativa, temperatura y viento desfavorables.

Tanto para el agua como para el adyuvante, el aumento de presión de trabajo resultó en la reducción de la uniformidad del espectro de gotas. Con relación a las presiones en los diferentes caldos, sólo la presión de $300 \mathrm{kPa}$ presentó diferencia, siendo el caldo con adyuvante el que presentó mayor uniformidad.

\section{LITERATURA CITADA}

BAUER, F. C.; RAETANO, C. G. Distribuição volumétrica de calda produzidas pelas pontas pulverização XR, TP e TJ sob diferentes condições operacionais. Planta Daninha, v. 22, n. 2 , p. $275-284,2004$

Planta Daninha, Viçosa-MG, v. 28, n. 4, p. 897-905, 2010
BAUER, F. C.; RAETANO, C. G.; PEREIRA, F. D. A. R. Padrões de distribuição volumétrica de pontas de pulverização de jato plano 11002, com e sem indução de ar, sob diferentes espaçamentos e alturas. Eng. Agric., v. 26, n. 2, p. 546-551, 2006.

CAMARA, F. T. et al. Distribuição volumétrica e espectro de gotas de bicos hidráulicos de jato plano de faixa expandida XR11003. Eng. Agríc., v. 28, n. 4, p. 740-749. 2008.

CUNHA, J. P. A. R.; TEIXEIRA, M. M; FERNANDES, H. C. Avaliação do espectro de gotas de pontas de pulverização hidráulicas utilizando a técnica da difração do raio laser. Eng. Agric., v. 27, p. 10-15, 2007. (Edição Especial).

CUNHA, J. P. A. R. et al. Uniformidade de distribuição de líquido por bicos de pulverização hidráulicos de jato plano e de jato cônico vazio. Eng. Agric., v. 12, n. 3, p. 192-202, 2004

FAO. FOOD AGRICULTURE AND ORGANIZATION. Equipo de aplicación de pesticida para uso en agricultura: equipo impulsado mecánicamente. Roma: 1997. 150 p. v. 2.

FERNANDES, A. P. et al. Caracterização do perfil de deposição e do diâmetro de gotas e otimização do espaçamento entre bicos na barra de pulverização. Eng. Agric., v. 27, n. 3, p. 728-733, 2007.

FERREIRA, M. C. et al. Fatores qualitativos da ponta de energia hidráulica ADGA 110015 para pulverização agrícola. Eng. Agric., v. 27, n. 2, p. 471-478, 2007.

FREITAS, F. C. L. et al. Distribuição volumétrica de pontas de pulverização Turbo Teejet 11002 em diferentes condições operacionais. Planta Daninha, v. 23, n. 1, p. 161-167, 2005. 
GALLI, J. C.; MATUO, T.; SIQUEIRA, E. C. Padrão de distribuição de alguns bicos hidráulicos. Planta Daninha, v. 6, n. 2, p. $144-150,1983$.

KUVA, M. A. et al. Períodos de interferência das plantas daninhas na cultura da cana-de-açúcar. III - capim-braquiária (Brachiaria decumbens) e capim-colonião (Panicum maximum). Planta Daninha, v. 21, n. 1, p. 37-44, 2003

NATION, H. J. The dinamic behaviour of field sprayer booms. J. Agric. Eng. Res., v. 36, n. 1, p. 61-70, 1982.

MATUO, T. Técnicas de aplicação de defensivos agrícolas. Jaboticabal: FUNEP, 1990.

PERECIN, D.; PERESSIN, V. A. Avaliação do padrão de distribuição de bicos para aplicação de herbicidas: efeitos da altura do alvo nos padrões de distribuição. Bragantia, v. 62, n. 3 , p. $477-97,2003$
RAMOS, H. et al. Manual de tecnologia de aplicação de produtos fitossanitários. São Paulo: ANDEF, 2006.

SPRAYING SYSTEMS. Teejet spray products: guia do comprador 202-BR/P. Wheaton: 2003. 37 p.

TEEJET. Boquillas para aplicación al voleo. Wheaton: Spraying systems, 2008. 192 p.

VIANA, R. G. et al. Características técnicas de ponta de pulverização LA-1JC e SR-1. Planta Daninha, v. 25, n. 1, p. 211-218, 2007.

WOLF, D. D.; SMITH, E. S. Uniformity of seed and fertilizer distribution with a hand-operated spinning spreader. Trans. Am. Soc. Agron. Eng., v. 22, n. 4, p. 761-762, 1979. 